\title{
Controlling the Expansion into Vacuum-the Enabling Technology for Trapping Atmosphere-Sampled Particulate Ions
}

\author{
Hideya Koizumi, ${ }^{a}$ Xiaoliang Wang, ${ }^{\mathrm{b}}$ William B. Whitten, ${ }^{\mathrm{a}}$ and \\ Peter T. A. Reilly ${ }^{a}$ \\ ${ }^{a}$ Oak Ridge National Laboratory, Oak Ridge, Tennessee, USA \\ ${ }^{\mathrm{b}}$ Department of Mechanical Engineering, University of Minnesota, Minneapolis, Minnesota, USA
}

\begin{abstract}
A new inlet has been designed to control the kinetic energy distributions of ions into a large-radius, frequency-adjusted, linear quadrupole ion trap. The work presented here demonstrates trapping singly-charged, intact proteins in the 10 to $200 \mathrm{kDa}$ range injected from the atmosphere. The trapped ions were held while collisions with a buffer gas removed the remaining amounts of expansion-induced kinetic energy. The ions were then ejected from the trap on-demand into an awaiting detector. There is no low mass limit for ion injection and trapping. The upper limit presented in this study was defined by the limit of the conversion dynode-based detector at $\sim 1.5 \mathrm{MDa}$. Trapping larger masses should be achievable. The transmission and capture efficiency across the entire mass range should be very high because the entire flow from the inlet empties directly into the trap. The kinetic energy distribution of massive ions is the primary reason for the working range limitation of mass spectrometers. Trapping ions with collisional cooling before mass analysis permits the motion of the ions to be completely defined by the applied fields. For this reason, this new inlet and trapping system represents a large step toward sensitive, high-resolution mass spectrometry into the megadalton range and beyond. (J Am Soc Mass Spectrom 2010, 21, 242-248) (C 2010 American Society for Mass Spectrometry
\end{abstract}

\section{A} rguably, the greatest advances in mass spectrometry of large molecules came with the introduction and development of electrospray ionization (ESI) [1] and matrix-assisted laser desorption ionization (MALDI) [2]. The creation of massive ions in vacuum was the start of a revolution in biological analysis using mass spectrometry. Unfortunately, the molecular weight range of mass specific biological species, such as proteins, RNA, DNA, and even viruses was much larger than the mass range of the mass spectrometer from which useful information could be obtained. Yet, the information was so valuable that a multitude of techniques and an entire industry was developed over the past 20-plus years so that these biological species could be analyzed using mass spectrometry. The essential thrust of the advances (beyond ionization) that have permitted the use of mass spectrometry for biological analysis has been in areas of sample preparation and analysis techniques, which allow analytes that are larger than the range of the mass spectrometer to be characterized with it anyway.

Address reprint requests to Dr. P. T. A. Reilly, Oak Ridge National Laboratory, Laser Spectroscopy and Micro Group, P.O. Box 2008, MS 6142, Oak Ridge, TN 37831, USA. E-mail: ReillyPT@ornl.gov
The thrust of our efforts has been to increase the range of mass spectrometers where they achieve good resolution, mass accuracy, and sensitivity. Our analysis suggested that the overarching reason for the limitation of mass spectrometers in general was the expansioninduced kinetic energy distribution of the ions as they are injected in the mass spectrometer. The average and spread of the kinetic energy distribution of the ions expanding into vacuum monotonically increases with increasing mass. It is not difficult to compensate for the average increase for a particular type of ion; however, it is extremely difficult to compensate for the increase in the spread of kinetic energies.

Indeed, it is the spread of the initial kinetic energy distribution that degrades resolution and sensitivity as a function of increasing mass for all forms of mass spectrometry. This is most obvious for MALDI timeof-flight (TOF) mass spectrometry, where the widths of the mass peaks are observed to increase with increasing mass [3]. It can also be observed in ESI of complexes where much more massive complex ions exhibit much broader peak widths than a corresponding monomer with the equivalent mass-to-charge ratio and isotope spread [4]. In both cases, the expansioninduced kinetic energy distribution is defined by the mass of the ion, not the mass-to-charge ratio. The spread of this 
distribution greatly contributes to the spread of flight times and, hence, the loss of resolution.

The only way that we have found to resolve this issue is to eliminate the expansion-induced kinetic energy. Trapping the ions and letting them translationally cool in the presence of a buffer gas presents a possible path forward-if they can be trapped. For example, Chernushevich and Thomson [5] collisionally cooled and trapped $692 \mathrm{kDa}$ proteosome $20 \mathrm{~S}$ ions in a linear trap at elevated pressure ( $\leq 38$ mTorr). Their work showed that even particulate ions (i.e., massive ions) can be trapped and mass-analyzed. We point out that their experiments were carried out on multiplycharged ions with a maximum mass-to-charge ratio of $\sim 34 \mathrm{kDa}$. If they had carried out their experiments with singly-charged ions of the same mass, they would have to compensate for the increased mass-to-charge ratio by reducing the frequency of their quadrupole, thereby limiting the lower end of their trapping range.

Since the advent of the digital ion trap [6], our group has proposed using aerodynamics to slow the forward momentum of massive ions so that they could be electrodynamically trapped. We knew that singlycharged massive ions could be trapped if the final expansion into vacuum could be controlled so that the kinetic energy of the injected ions would not be significantly greater than and preferably less than the pseudopotential well depth of the trap [7]. The development of the hardware that permits the digital generation of trapping potentials whose frequencies can be rapidly changed [6] provided a method to optimize the well depth as a function of $m / z$ for any ion mass.

Because of the complexity of the complete effort, it was decided that the project for producing high-resolution mass spectra in the ultra high mass range $(>20 \mathrm{kDa})$ would be broken into separate segments. The first two segments involved the proof of principle for trapping massive singly charged ions. From our work with aerodynamic lens inlet systems [8], we knew that the kinetic energy induced through a mere 2-Torr supersonic expansion into a vacuum in the mTorr pressure range or lower was shown to be significant and capable of thwarting trapping of ions in the $100 \mathrm{kDa}$ mass range. Consequently, the pressure of the final supersonic expansion into vacuum should be less than 2 Torr and adjustable if possible.

However, controlling the final expansion into vacuum would create a dispersive flux of injected ions. For this reason, our group designed a large-radius, frequencyadjusted linear quadrupole ion trap [8] (LR-LQIT) that is capable of accepting dispersive injection of ions. The basic idea was to markedly increase the radius of a linear ion trap to increase the trapping efficiency of ions with relatively large off-axis velocity components. We used the LR-LQIT to capture singly-charged bovine serum albumin (BSA) ions $(\mathrm{m} / z=66,000)$. In that publication, we also used the trapping frequency to demonstrate that only singly charged $\mathrm{BSA}^{+}$ions were trapped. To increase the trapping range of our trap, we had to decrease the kinetic energy of the ions injected into to it.

In this publication, a method for controlling the final expansion into our frequency-adjusted, large-radius linear quadrupole ion trap is revealed. Trapping of singlycharged proteins up to myosin at $m / z=205 \mathrm{kDa}$ is demonstrated. Larger particulate ions up to $1.5 \mathrm{MDa}$ have been studied with this inlet. Unfortunately, our current detection system set the upper limit and prohibited the determination of the true trapping limit for the combination of our inlet and trap. For now, we can only conclude that the combination can trap ions up to $m / z=1.5 \mathrm{MDa}$.

\section{Design of an Inlet for Controlling the Kinetic Energies of Injected Ions}

Our inlet permits particulate ions at atmospheric pressure to be introduced into vacuum and trapped in a gas-filled linear quadrupole ion trap for subsequent on-demand injection into a mass spectrometer. This inlet is based on the principle of using an opposing jet to reduce the forward momentum of the expanding particles. A schematic of the inlet is shown in Figure 1a. Particulate ions are expanded through the flow-limiting inlet orifice where they are entrained in a laminar flow of carrier gas (air) at reduced pressure. They flow into a plenum chamber, where they eventually exit through a final expansion into a gas-filled linear ion trap. The final expansion is a key piece of technology that is based on using a counter-propagating jet of gas to slow the particulate ions after expansion-induced acceleration. The final expansion nozzle is shown in Figure 1b. It consists of a $1.5 \mathrm{~cm}$ diameter cylinder positioned in
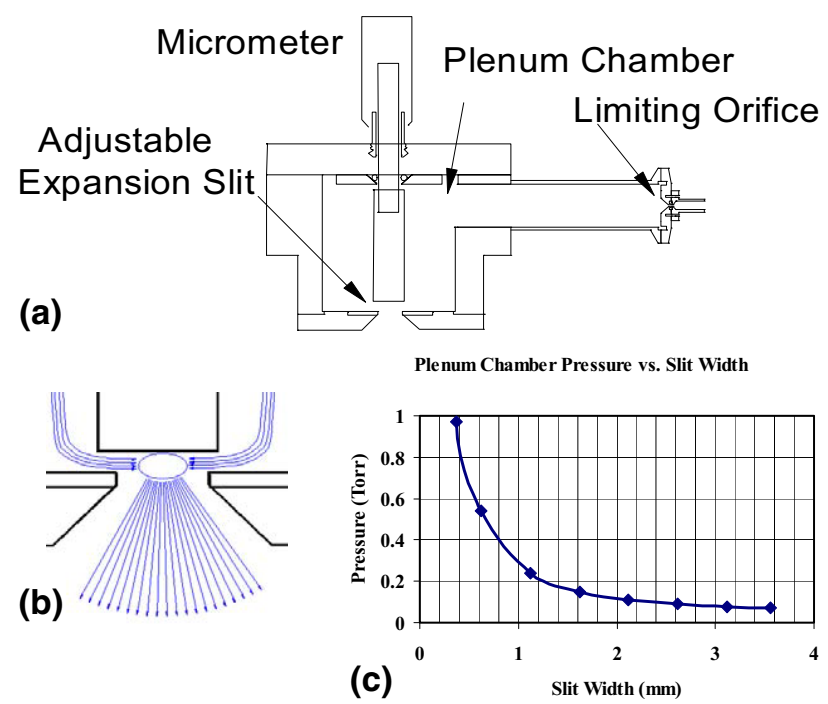

Figure 1. (a) Schematic of the inlet for controlling the final expansion into vacuum. (b) Depiction of the flow contours of the particulate ions during the final expansion. (c) Plot of the plenum chamber pressure as a function of cylinder/expansion orifice distance (slit width). These results are for a $10 \mathrm{~mm}$ expansion orifice and a $15 \mathrm{~mm}$ cylinder. 
front of an orifice. There were three options for the orifice diameter, $5,7.5$, and $10 \mathrm{~mm}$. There is a pressure drop between the plenum chamber and the quadrupole chamber. The reduced pressure aerosol in the plenum chamber flows through the chamber and eventually expands between the cylinder and the orifice plate. The expansion occurs inwardly with axial symmetry. Eventually, the dynamics of the expansion will cause the particulate ions to expand in the axial direction into the gas-filled quadrupole chamber (see Figure 1b). Because momentum transfer is dependent on the difference in velocity between the gas and the particles, particulate ions expanding from one side of the inward expansion cannot pass back into the plenum chamber and must either plate out on the walls or move out into the quadrupole chamber. The particulate ion velocity distribution into the gas-filled linear quadrupole ion trap depends primarily on the pressure differential between the plenum and quadrupole chambers and the orifice diameter. The pressure differential will change with the distance between the cylinder and the orifice. In our design, the cylinder is mounted on a micrometer so that this distance can be changed while under vacuum. The pressure in the plenum chamber can be incrementally adjusted from 70 mTorr to essentially atmospheric pressure for the $10 \mathrm{~mm}$ diameter orifice. A plot of the plenum chamber pressure as a function of slit width for a $10-\mathrm{mm}$ orifice is shown in Figure 1c. This proves that the pressure of the final expansion into the quadrupole chamber can be controlled. By controlling the pressure of the expansion, we can adjust the probability of stopping and capturing the particulate ions in the gas-filled linear quadrupole ion trap.

\section{Experimental}

The particulate ions used in this study were generated by a variety of methods. Two commercial aerosol generators were used. An electrospray aerosol generator (TSI, Inc., Shoreview, MN, USA, model 3480) was used to produce singly-charged aerosols from the proteins used in this study. Nanoparticles of urea were created by nebulizing and drying a urea solution. The solvent was 1:1 ratio of methanol to water. The urea aerosol was size selected with an electrostatic classifier (TSI, Inc., model $3085 \mathrm{~N}$ ). The output of the electrostatic classifier is monodisperse (having a narrow size distribution) and primarily singly-charged. Attempts were also made at increasing the charged ion output using desorption electrospray ionization (DESI). In this procedure, protein solutions were electrosprayed at a flat surface near the entrance orifice. The multiply-charged droplets impacted the surface and bounced off transferring most of their charge, leaving primarily singlycharged protein ions. The rapid loss of charge on the droplet results from the electrical/chemical potential between the charged droplet and the grounded metal surface. The final charge distribution was found to be very dependent on the angle of incidence. Grazing incidence angles produced higher average charge distributions.

The output of the inlet was examined with two different digitally operated quadrupoles. In the first experiments, the inlet output was examined with a quadrupole with $0.635 \mathrm{~cm}$ diameter, $34.5 \mathrm{~cm}$ long rods. The quadrupole radius was $0.277 \mathrm{~cm}$. This quadrupole was operated as a mass filter by varying the duty cycle of the square waveform [9]. The duty cycle was varied from 0.5 to 0.4 to obtain the best resolution while maintaining sufficient throughput to clearly observe a spectrum [10]. The low voltage waveforms were generated with a digital to analog converter (DAC) (National Instruments, Austin, TX, USA, PCI-6120). The low voltage square waveforms were then input into high voltage DC pulsers (DEI, Vista, CA, USA, PVX4150). The voltage of the waveforms was varied from 250 to $500 \mathrm{~V}_{0-p}$.

The second quadrupole configuration used to examine the inlet was a quadrupole with a $5.42 \mathrm{~cm}$ radius [8]. This design uses a circularly concave electrode structure to minimize the cross section of the device while maximizing the radius. The concept of using this structure was first demonstrated by Hayashi and Sakudo [11] and later illustrated in Dawson's text [10]. This configuration was used to create a large-radius linear quadrupole ion trap (LR-LQIT). The operation of the LR-LQIT was demonstrated in an earlier publication [8]. In these experiments, the ions were collected, held in a 5-mTorr buffer gas (air), and then ejected ondemand into a conversion dynode/channeltron detector. This quadrupole was not used as a mass filter; therefore the duty cycle of the waveforms was always set to 0.5 . The square waveforms were generated with a function generator (Stanford Research Systems, Sunnyvale, CA, USA, DS335 $3 \mathrm{MHz}$ ) and high voltage pulsers (DEI, PVX-4150). The voltage of the waveforms was varied from 250 to $700 \mathrm{~V}_{0-p}$. In both experiments, the conversion dynode was operated between 10 and $20 \mathrm{KV}$.

\section{Results and Discussion}

We first examined the inlet by using a linear quadrupole with $0.635 \mathrm{~cm}$ diameter rods (see Figure 2). We operated the quadrupole as a mass filter by varying the duty cycle of the applied waveform. Changing the duty cycle is the equivalent of adding a DC component to the waveform [12]. Though the resolution was poor and the transmission was low, we were able to resolve the spectra of large molecules and particles up to $\sim 1.5 \mathrm{MDa}$. The resolved spectra of immunoglobulin $\mathrm{G}(\mathrm{IgG})$ and urea particles are shown in Figure 3. The assignment of the particle mass-to-charge ratio was confirmed by sampling the electrosprayed IgG into a TSI Inc. model $3085 \mathrm{~N}$ atmospheric pressure differential mobility analyzer with a condensation nuclei counter to detect the electrostatically classified particles. The ion mobility measurements showed that the majority of $\mathrm{IgG}$ ions produced were singly charged. The range of fre- 


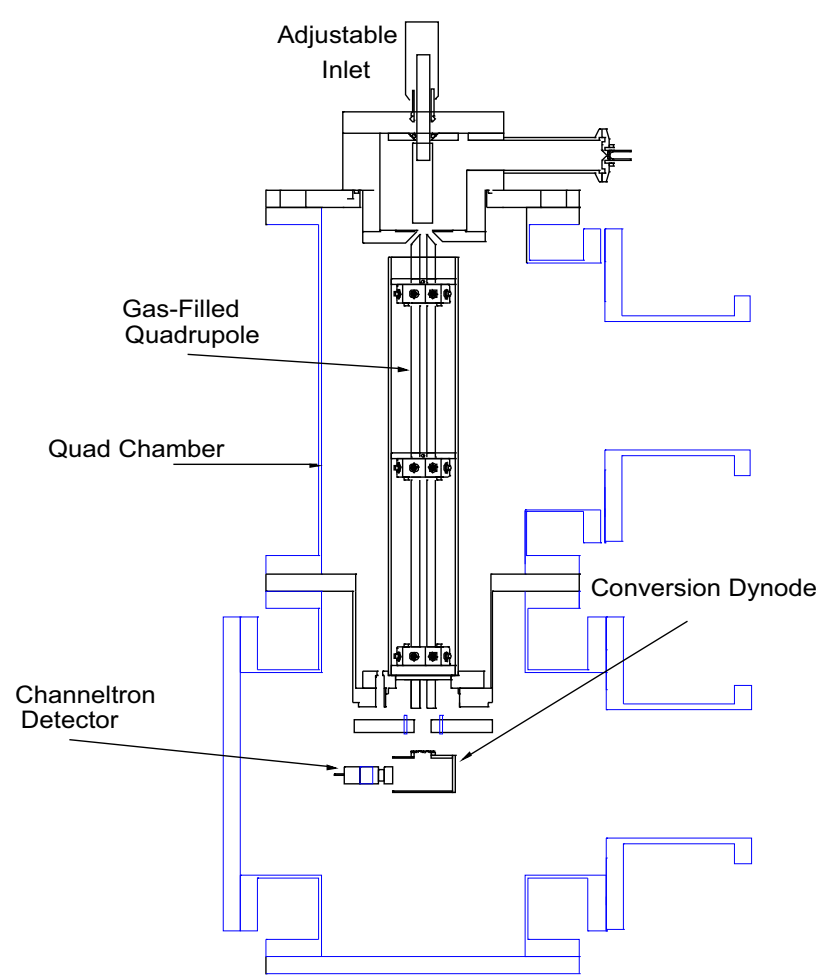

Figure 2. Test apparatus for the kinetic energy-reducing inlet. A quadrupole with $1 / 4$ in. diameter rods was digitally operated to control the flow of ions to the detector.

quencies that the IgG ions were transmitted through the quadrupole also defined the mass-to-charge ratio from the following equation:

$$
\frac{m}{z}=\frac{1000 e A_{N} V_{0-p}}{q_{x-y} r_{0}^{2} \pi^{2} f^{2}}
$$

where $e$ is the protons charge, $A_{N}$ is Avogadro's number, $V_{0-p}$ is the AC voltage zero to peak, $r_{0}$ is the distance between the central quadrupole axis and the rod surface, $f$ is the frequency of the potential and $q_{x-y}$ is the Mathieu parameter for a linear quadrupole. Because we use digitally-created square waves to drive the quadrupole, the apex of the stability diagram occurs near a Mathieu parameter of 0.6 rather than a value near 0.8 for a sinusoidal potential. The peak transmission of the IgG ions occurred near $65 \mathrm{kHz}$. They were not multiply charged. The mass range analyzed was limited to less than 1.5 MDa by the conversion dynode/channeltron detector. Larger particulate ions were easily generated with the electrostatic classifier; however, they did not yield a signal.

Consider singly-charged immunoglobulin $G$ ions coming out of a 2-Torr supersonic expansion into vacuum. The velocity of the ions in vacuum would be roughly $400 \mathrm{~m} / \mathrm{s}$, yielding $90 \mathrm{eV}$ of kinetic energy [13]. If the quadrupole mass filter was operating at $80 \mathrm{kHz}$, the unslowed ions would experience 60 cycles of the trap. According to Dawson [10], the limiting resolution is defined by:

$$
(\mathrm{m} / \Delta \mathrm{m})=\mathrm{N}^{\mathrm{n}} / \mathrm{K}
$$

where the $\mathrm{N}$ is the number of cycles of the rf field and $\mathrm{K}=20$ and $n=2$ (experimentally determined parameters). Consequently, the best resolution that would be achieved under those conditions would be $(\mathrm{m} / \Delta \mathrm{m})=$ 180 . Our result with IgG yields a resolution of roughly $(\mathrm{m} / \Delta \mathrm{m})=20$. The pressure of the expansion where IgG ions were mass analyzed was more than an order of magnitude less than the 2-Torr supersonic expansion from which the ion velocities were calculated. Our measured IgG ions were moving much slower through the quadrupole and therefore experiencing more than 60 cycles. We therefore believe that our measurements are valid even though they were performed in a gasfilled quadrupole. Furthermore, we felt confident that our inlet and trap were working well together to deliver slow moving massive ions to the detector because biasing the exit endcap electrode by $10-20 \mathrm{~V}$ over the entire range stopped the flow of ions to the detector. Unfortunately, we were still unable to trap any large ions in the quadrupole for subsequent ejection. Apparently, the ions escaped before they could be ejected into the detector.

Trapping the large masses for subsequent mass analysis had always been the goal of our work. It was for this reason that we set out to build a better ion trap. We assumed that the inability to trap the massive ions resulted because the fringe fields from the end of the electrodes penetrated too far into the quadrupole so
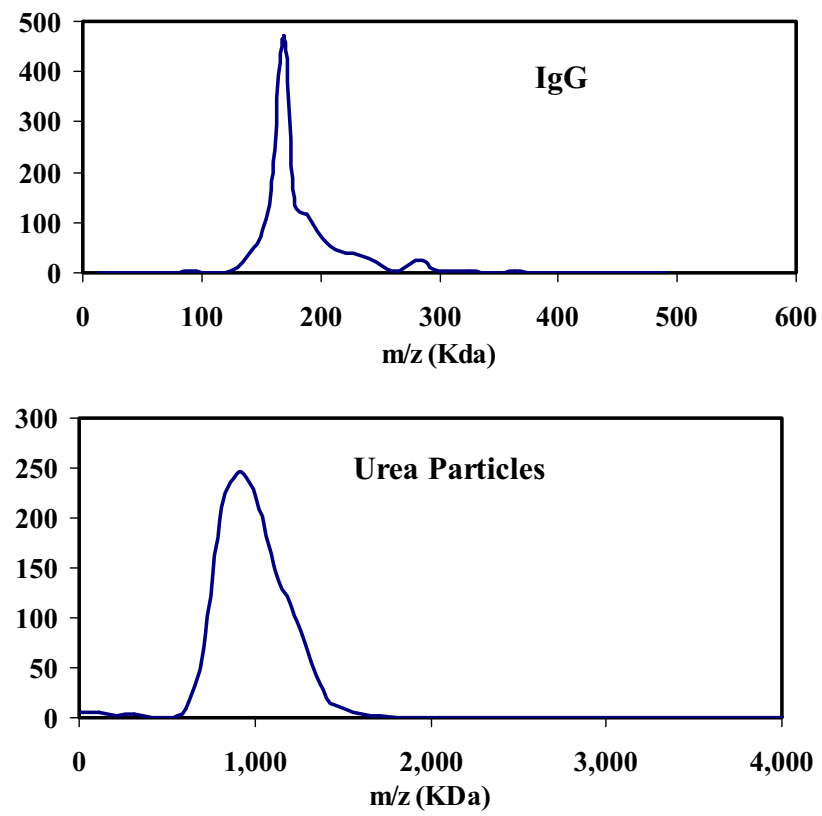

Figure 3. Mass analysis of immunoglobulin $\mathrm{G}$ and urea particles with the kinetic energy reducing inlet and operation of the quadrupole as a mass filter. 
that the massive ions had a path out of the trap as they settled in the quadrupole. We also knew that the expansion of the ions into the quadrupole was dispersive so the probability of entraining the big ions into the narrow quadrupole aperture was small. For these reasons, we thought that a quadrupole with a larger radius would work better and we set out to increase the quadrupole radius by at least a factor of 10 . The illustration of circular concave electrodes to create hyperbolic fields found in Dawson's text inspired our large radius design. The LR-LQIT was first demonstrated in a previous publication using singly-charged bovine serum albumin ions and an aerodynamic lens inlet system [8].

A schematic of the new inlet integrated with our large radius trapping system is shown in Figure 4. The entire atmospheric input to the inlet empties directly into the LR-LQIT. The pressure inside the large radius trap is maintained at $\sim 5 \mathrm{mTorr}$ (air) while the chamber was pumped with a $255 \mathrm{~L} / \mathrm{s}$ turbo pump (Edwards Vacuum, Tewksbury, MA, USA). The system was oriented in the vertical direction as depicted in the schematic so that the force of gravity acted along the axis of the quadrupole trap.

The inlet and LR-LQIT were initially tested with bovine serum albumin (BSA) at $66 \mathrm{kDa}$. The electrospraygenerated aerosol was directed into $100-\mu \mathrm{m}$ diameter flow limiting orifice. Ions were detected without added buffer gas in the chamber or energizing the LRLQIT. The pressure in the quadrupole chamber was 5

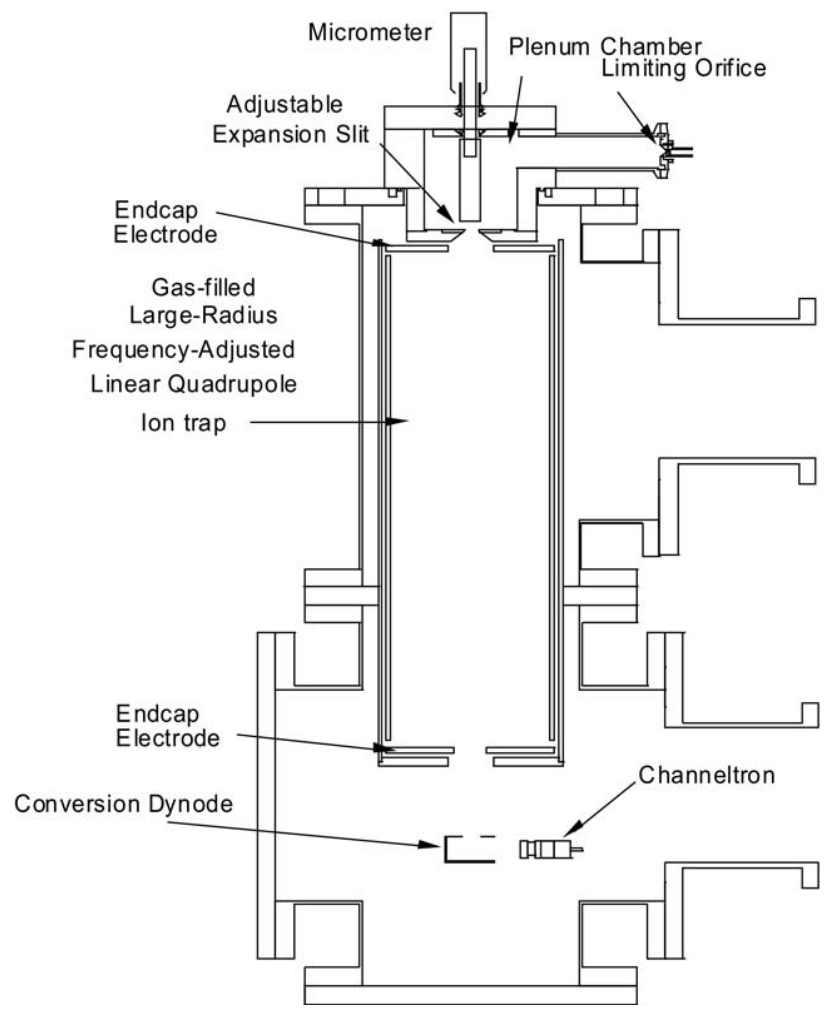

Figure 4. Schematic of the new inward-expansion inlet and large-radius, linear quadrupole ions trap test apparatus.
mTorr. Energizing the LR-LQIT $\left(700 \mathrm{~V}_{0-p}, 8 \mathrm{kHz}\right)$ with grounded endcap electrodes stopped the flow of ions to the detector at any plenum chamber pressure. At this point, it was recognized that the inlet was probably working too well. The kinetic energy of the singlycharged BSA ions was not high enough to penetrate the potential barrier created by the penetration of the quadrupole fields through the hole in the entrance endcap electrode.

The orifice diameter was then changed to $5 \mathrm{~mm}$ to increase the kinetic energy of the BSA ions and the experiment was tried again. This time, when the LRLQIT was energized, the ion yield increased. Applying a $25 \mathrm{~V}$ or greater bias to the entrance endcap electrode stopped the flow of ions into the LR-LQIT suggesting that the kinetic energy of the BSA ions was less than $25 \mathrm{eV}$. Twenty and $100 \mathrm{~V}$ biases were applied to the entrance and exit endcap electrodes, respectively, to trap the ions. Singly-charged protein ions and mixtures in the range between 10 and $205 \mathrm{kDa}$ were trapped and detected with this test set up. The mass-to-charge ratios of the trapped ions were confirmed by adjusting the frequency of the trap so that multiply-charged $(+2$ or higher) or low mass ions could not be trapped by using eq 1 . Changing the frequency does not permit definition of the mass-to-charge ratios with great accuracy, but it is easy to roughly define $\mathrm{m} / \mathrm{z}$. Precise definition of the mass came from knowing the analyte injected. While it is true that we cannot tell with absolute certainty that small fragments of the proteins were not lost in the injection and trapping process, we do know that masses trapped had some preset minimum. For example, we set the minimum trapping mass in the BSA experiments to $51 \mathrm{kDa}$ by adjusting the square waveform to $8 \mathrm{kHz}$ at $700 \mathrm{~V}_{0-p}$ (eq 1). Under these conditions, smaller ions than $m / z-51,000$ have Mathieu parameters $\left(q_{z}\right)$ greater than 0.7125 and they are therefore not stable in the trap. Similar checks were conducted for each mass trapped. Therefore, we are absolutely certain that we were injecting and trapping singly-charged massive ions over the entire range.

Examples of the detector profiles for singly-charged proteins and protein mixtures are shown in Figure 5. The onset of detection changed modestly with mass after the endcap electrode potential was dropped. However, the overall temporal profile did not change significantly as a function of mass for this test setup. Previously, we reported observation of changes in the arrival time of the trapped ions at the detector as a function of analyte mass when they were ejected from the LR-LQIT [8]. That was not observed in this case because the entire flow from the inlet exits directly into the LRLQIT, whereas in the previous study the aerodynamic lens system inlet was differentially pumped to remove the carrier gas. In these experiments the net flow of carrier gas through the LR-LQIT keeps the ions from separating as a function of ion mobility when they are ejected as they did in our previous publication [8]. This inlet has also been used for analyzing nanoparticles of 

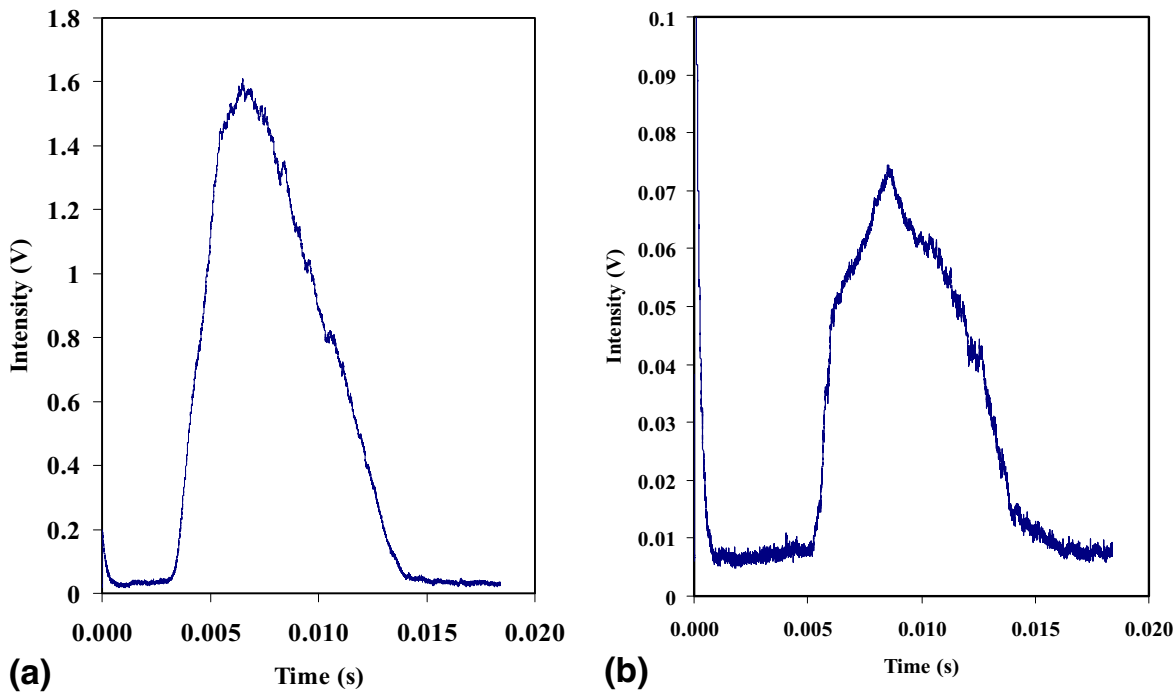

Figure 5. Examples of the detector time response of trapped ions ejected from the LR-LQIT after dropping the exit end cap potential. (a) Ejection profile of a mixture of singly-charged proteins at 14.2, 20.1, 24.0, 29.0, 36.0, 45.0, and $66.0 \mathrm{kDa}$ (Dalton Mark, Sigma Aldrich). (b) Ejection profile of singly-charged Myosin (205 kDa).

urea. They yield ejection profiles that were essentially indistinguishable from the profiles shown in Figure 5. Because they all looked roughly the same, we have not included ejection profiles of each analyte trapped. However, we applied the low enough trapping frequencies for each analyte to be certain that multiply-charged analyte and small ions did not remain trapped.

Our observed trapping limit was not due to limitations in the ability of the inlet and LR-LQIT to trap the massive singly-charged ions rather it was due to the limitations of the detector. The upper limit of the detector was previously established using an aerodynamic lens based inlet without the LR-LQIT energized. We also observed the detector limit using the small quadrupole as a mass filter.

The work presented here conclusively shows that singly-charged proteins and protein complexes can be trapped and held so that all of the expansion-induced kinetic energy is removed from the ions. The trapped ions could then be ejected on-demand from the LRLQIT into an awaiting mass analyzer. The kinetic energy distribution of the ions injected into a mass analyzer from our inlet and trapping system would only be defined by the applied fields.

The sensitivity of our inlet and trapping system should also be extraordinary. The particle transmission efficiency through the plenum chamber should be roughly as good as the transmission efficiency through an aerodynamic lens system given the similar flow patterns (see Figure $1 \mathrm{~b}$ and reference [13] for a comparison). The transmission efficiency for aerodynamic lens systems is generally said to be $100 \%$ for the range of sizes for which it was designed [14]. There will be an upper mass limit for plenum transmission just as there is for transmission through an aerodynamic lens system [14] that is based on the stopping distance of the particles $[8,15]$. For a $1-\mu \mathrm{m}$ diameter particle traveling at $500 \mathrm{~m} / \mathrm{s}$ through a 2 Torr gas, the stopping distance is $\sim 13 \mathrm{~cm}$. Therefore, $13 \mathrm{~cm}$ of travel is required to entrain $1 \mu \mathrm{m}$ particles in the laminar flow in the chamber. This is a maximum stopping distance because the more massive the particles, the greater the difference in velocity between the expanding gas and the particle. Smaller particles will be entrained in shorter distances. $200 \mathrm{~nm}\left(\sim 10^{9} \mathrm{Da}\right)$ particles should make through the plenum chamber with ease under a wide range of pressures. The greatest losses to sensitivity should occur during the first expansion and passage through the plenum chamber and result from neutralization of the charged particles mainly by interaction with a surface. The most likely place for this to occur is during the final expansion into the LR-LQIT. It is our contention that the surface interaction during the final expansion should be much less than that which occurs by passage through a capillary for example. Therefore, sensitivity loss by neutralization should be at least as good or better than any other type of atmospheric inlet. Finally, the entire flow from the flow limiting atmospheric inlet orifice passes directly through the entrance endcap into the LR-LQIT. The forward momentum of the injected ions is stopped by collisions with the buffer gas inside the quadrupole before they can reach the exit plane of the trap. Consequently, ions can be captured by the LR-LQIT even when the well depth is modest. The ions are separated from the carrier gas inside the LR-LQIT after they are already trapped. Our design eliminates ion loss during the rough pumping stage and limits surface-induced neutralization. We expect that our greatest ion loss will occur during the transfer process between the LR-LQIT and the mass analyzer. Therefore, we expect to eventually obtain much better 
sensitivity than currently available atmospheric inletbased mass spectrometers.

The upper mass limit of the inlet and trapping system has yet to be determined. Larger particulate ions will require higher plenum chamber pressures for efficient expansion into the LR-LQIT. At 250 mTorr, a 200 $\mathrm{nm}\left(10^{9} \mathrm{Da}\right)$ particle requires a maximum stopping distance of $\sim 20 \mathrm{~cm}$. Doubling the pressure halves the stopping distance. Higher plenum chamber pressures can still yield low enough kinetic energy distributions because of the design of the final expansion. The expansion from the plenum chamber into the quadrupole chamber is axially symmetric. Gases and particulate ions initially expand toward the symmetry axis where they meet, mix and then undergo a secondary expansion in the perpendicular direction along the symmetry axis out into the quadrupole. The secondary expansion has an even lower pressure drop that yields slow moving ions that can then be trapped in the LR-LQIT. The secondary and final expansion into the quadrupole chamber can be optimized with the size of the final expansion orifice and the slit width. It is our contention that even particulate ions in the gigadalton range can be delivered into the LR-LQIT and trapped with great efficiency with the proper selection of the orifice and slit width dimensions.

Trapping of the large particulate ions over the entire range below $1.5 \mathrm{MDa}$ was accomplished with the $5-\mathrm{mm}$ orifice and a plenum chamber pressure at $\sim 250$ mTorr. We did not work to optimize the trapping efficiency. We propose that optimization of the trapping efficiency of our system will be best done when a detector is in place that can extend the mass range or with fluid dynamics calculations. Our work suggests that unprecedented sensitivity may be achieved. We propose that our current version of the inlet and trapping system should enable sensitive atmospheric pressure MALDI at essentially any mass below $1 \mathrm{MDa}$.

Finally, trapping ions before mass analysis allows their motion to be completely defined by the applied fields. We suggest that this work represents the first step toward high-resolution mass analysis between 20 $\mathrm{kDa}$ and 1.5 MDa or higher. Our future work will involve coupling our new technology to mass analyzers that will provide sensitive, high-resolution mass analysis in the ultrahigh mass range.

\section{Conclusions}

We have demonstrated that the combination of our new inlet and trapping system can trap and hold massive ions up to 1.5 MDa in mass. These ions can be delivered on-demand to an awaiting mass analyzer with a kinetic energy distribution that is defined by the applied fields. Trapping massive ions is an important step toward high-resolution mass analysis in the ultrahigh mass range above $20 \mathrm{kDa}$.
The upper mass limit of our device is currently defined by the limit of our conversion dynode-based detector system ( 1.5 MDa). Our inlet and trapping system has no lower mass limit and as yet an undefined upper mass limit greater than 1.5 MDa. Future experiments are planned to define this limit.

All of the work done here has been performed with the goal of extending the working range of mass spectrometers up into the gigadalton range. The next step in the proposed evolution is to begin resolved mass measurements up into the megadalton range. There are two types of mass spectrometers that can accommodate singly-charged massive ions while providing highresolution, time-of-flight and digital ion traps. We plan to use our trapping system as an ion source for these types of spectrometers to provide high-resolution measurements of intact singly-charged proteins and protein complexes soon.

\section{Acknowledgments}

The authors acknowledge support for this research by maturation funding from UT-Battelle, LLC under contract no. DE-AC0500OR22725 with Oak Ridge National Laboratory, managed and operated by UT-Battelle, LLC.

\section{References}

1. Fenn, J. B.; Mann, M.; Meng, C. K.; Wong, S. F.; Whitehouse, C. M Electrospray Ionization for Mass-Spectrometry of Large Biomolecules. Science 1989, 246, 64-71.

2. Karas, M.; Hillenkamp, F. Laser Desorption Ionization Of Proteins with Molecular Masses Exceeding 10,000 Daltons. Anal. Chem. 1988, 60, 2299-2301.

3. Wenzel, R. J.; Matter, U.; Schultheis, L.; Zenobi, R. Analysis of Megadalton Ions Using Cryodetection MALDI Time-of-Flight Mass Spectrometry. Anal. Chem. 2005, 77, 4329-4337.

4. Harmer, N. J.; Robinson, C. J.; Adam, L. E.; Ilag, L. L.; Robinson, C. V. Gallagher, J. T.; Blundell, T. L. Multimers of the Fibroblast Growth Factor (FGF)-FGF Receptor-Saccharide Complex are Formed on Long Oligomers of Heparin. Biochem. J. 2006, 393, 741-748.

5. Chernushevich, I. V.; Thomson, B. A. Collisional Cooling of Large Ions in Electrospray Mass Spectrometry. Anal. Chem. 2004, 76, 1754-1760.

6. Ding, L.; Gelsthrope, A.; Nuttall, J.; Kumashiro, S. Proceedings 49th American Society for Mass Spectrometry Conference on Mass Spectrometry and Applied Topics; Chicago, Illinois, May 27-31, 2001

7. Dehmelt, H. G. Radiofrequency Spectroscopy of Stored Ions. Adv. At. Mol. Phys. 1967, 3, 53-72.

8. Koizumi, H.; Whitten, W. B.; Reilly, P. T. A. Trapping of Intact, Singly-Charged, Bovine Serum Albumin Ions Injected from the Atmosphere with a 10-cm Diameter, Frequency-Adjusted Linear Quadrupole Ion Trap. J. Am. Soc. Mass Spectrom. 2008, 19, 1942-1947.

9. Richards, J. A.; Huey, R. M.; Hiller, J. A New Operating Mode for the Quadrupole Mass Filter. Int. J. Mass Spectrom. Ion Phys. 1973, 12, 317-339.

10. Austin, W. E.; Holme, A. E.; Leck, J. H. The Mass Filter: Design and Performance. In Quadrupole Mass Spectrometry and Its Applications, Dawson, P. H., Ed.; AIP Press: Woodbury, NY, 1995; pp 129-131.

11. Hayashi, T.; Sakudo, N. Proceedings of the International Conference on Mass Spectrometry; Kyoto, Japan 1969, p 263.

12. Berton, A.; Traldi, P.; Ding, L.; Brancia, F. L. Mapping the Stability Diagram of a Digital Ion Trap (DIT) Mass Spectrometer Varying the Duty Cycle of the Trapping Rectangular Waveform. J. Am. Soc. Mass Spectrom. 2008, 19, 620-625.

13. Liu, P.; Ziemann, P. J.; Kittelson, D. B.; McMurry, P. H. Generating Particle Beams of Controlled Dimensions and Divergence. 2. Experimental Evaluation of Particle Motion in Aerodynamic Lenses and Nozzle Expansions. Aerosol Sci. Technol. 1995, 22, 314-324.

14. Liu, P.; Ziemann, P. J.; Kittelson, D. B.; McMurry, P. H. Generating Particle Beams of Controlled Dimensions and Divergence. 1. Theory of Particle Motion in Aerodynamic Lenses and Nozzle Expansions. Aerosol. Sci. Technol. 1995, 22, 293-313.

15. Friedlander, S. K. Smoke, Dust and Haze; John Wiley and Sons, Inc.: New York, 1977; p 96. 